\title{
OBSERVACIONES ECOLÓGICAS, COROLÓGICAS Y TAXONÓMICAS DE HÍDRIDOS DULCEACUÍCOLAS (CNIDARIA, HYDROZOA: HYDRIDAE) EN LA CUENCA DEL DUERO
}

\author{
Javier Morales $^{1 *}$, Ana I. Negro ${ }^{2} \&$ Miguel Lizana $^{1}$ \\ ${ }_{1}^{1}$ Área de Biología Animal. Universidad de Salamanca. E-37007. Salamanca, Spain. \\ iD (JM): https://orcid.org/0000-0002-7063-563X \\ iD (ML): https://orcid.org/0000-0002-4139-8639 \\ 2 Área de Ecología. Universidad de Salamanca. E-37007. Salamanca, Spain. \\ iD (AN): https://orcid.org/0000-0002-4541-6427 \\ *Autor para correspondencia: mormarja@usal.es
}

\section{RESUMEN}

Se describe la presencia de cuatro taxones de hidras de agua dulce (Cnidaria, Hydrozoa: Hydridae) en zonas someras epicontinentales de la cuenca del Duero (NO de España). En la subcuenca del Tera se encontraron individuos aislados sobre macrófitos sumergidos de tres taxones: Hydra vulgaris Pallas, 1766, Hydra (Pelmatohydra) oligactis Pallas, 1766 e Hydra (Chlorohydra) viridissima Pallas, 1766, y además en el Carrión grupos coloniales sobre bloques de cuarcita de la forma $H$. vulgaris var. aurantiaca Ehrenberg, 1838. En todos los casos se corresponde con ecosistemas de media montaña de aguas frías, transparentes y oligotróficas, de $\mathrm{pH}$ ligeramente ácido y con escasa mineralización. Todas las especies fueron localizadas en simpatría estricta, aunque en el lago de Sanabria se pudo citar tres taxones en diferentes hábitats. Se documenta la predación en cautividad de $\mathrm{H}$. oligactis sobre gusanos oligoquetos y los periodos de formación de pólipos por gemación.

Palabras clave: cnidarios dulceacuícolas; Hydridae; distribución; taxonomía; ríos y lagos; cuenca Duero.

\section{ABSTRACT}

Ecological, corological and taxonomic observations of freshwater hydrids (Cnidaria; Hydrozoa: Hydridae) in the Duero Basin.

The presence of four taxa of freshwater hydras (Cnidaria, Hydrozoa: Hydridae) is recorded in shallow epicontinental waters of the Duero basin (NW Spain). In the Tera subbasin, individuals were found isolated on submerged macrophytes of three taxa: Hydra vulgaris Pallas, 1766, Hydra (Pelmatohydra) oligactis Pallas, 1766 and Hydra (Chlorohydra) viridissima Pallas, 1766, and also in the Carrión colonial groups on quartzite blocks of the taxon $H$. vulgaris var. aurantiaca Ehrenberg, 1838. In all cases they inhabit medium altitude mountain ecosystems with cold, transparent and oligotrophic waters, and slightly acidic $\mathrm{pH}$ with low mineralization. All the species were found in strict sympatry, although the first three species in different habitats could be found in Lake of Sanabria. Predation in captivity of $\mathrm{H}$. oligactis on oligochaetes hookworms and periods of polyps incubation by budding are documented.

Key words: freshwater cnidaria; Hydridae; distribution; taxonomy; rivers and lakes; Duero basin; NW Spain.

Recibido/Received: 11/05/2018; Aceptado/Accepted: 10/09/2018; Publicado en línea/Published online: 16/10/2018

Cómo citar este artículo/Citation: Morales, J., Negro, A. I. \& Lizana, M. 2018. Observaciones ecológicas, corológicas y taxonómicas de hídridos dulceacuícolas (Cnidaria, Hydrozoa: Hydridae) en la Cuenca del Duero. Graellsia, 74(2): e077. https://doi.org/10.3989/graellsia.2018.v74.210

Copyright: (C) 2018 SAM y CSIC. This is an open-access article distributed under the terms of the Creative Commons Attribution 4.0 International (CC BY 4.0) License. 


\section{Introducción}

Distintos taxones del género Hydra Linnaeus, 1758 (Cnidaria; Hydrozoa) han sido ampliamente utilizados tanto para bioensayos de toxicidad, como en estudios de genética, histología y fisiología animal para comprender los procesos de regeneración de tejidos e incluso partes enteras de su cuerpo con simetría radial (Bode, 2003; Khalturin et al., 2008), hasta la total senescencia (Martínez \& Bridge, 2012). Sus rápidos ciclos de reproducción en cautividad (asexuales por gemación), su elevada y rápida tasa de regeneración, su plasticidad fenotípica y su facilidad de mantenimiento en laboratorio (Massaro \& Rocha 2008) han favorecido su popularización en este tipo de trabajos científicos.

Existen propuestas que utilizan las hidras en test de toxicidad ambiental (metales pesados y amoniaco sobre todo) para animales a través de su acción progresiva sobre los tejidos en concentraciones variables para conseguir efectos deletéreos o deformadores de tejidos, hasta los mortales por desarticulación de la estructura celular de los mismos (Beach \& Pascoe, 1998; Kar \& Aditya, 2007; Quinn et al., 2012; Mooney et al., 2018). Y por ello se utilizan, junto a un amplio grupo de invertebrados y peces, para establecer las Directrices de Calidad Ambiental para las aguas epicontinentales (CCME, 2010; USEPA, 2013). Asimismo el efecto de la exposición a radiaciones ionizantes que puede favorecer la formación de pies ectópicos en la base de las hidras ha sido estudiado para poder comprender los procesos de regeneración tisular de estas especies (Ghaskadbi et al., 2005; Stepanjants et al., 2006).

Sin embargo son pocos los trabajos en los que se relaciona la presencia de estos cnidarios dulceacuícolas con la tipología de los medios acuáticos y la calidad del agua (Huarachi \& González, 2012), más allá del efecto de la temperatura sobre la estrategia reproductora (sexual o gemación asexual) que es inducida por la evolución estacional de los ecosistemas acuáticos (Schroeder \& Callaghan, 1982; Kaliszewicz, 2011; Kaliszewicz \& Lipinska, 2013; Rosa et al., 2015; Tökölyi et al., 2016).

Es destacable que son un grupo poco estudiado hasta la actualidad y con escasas referencias en España. Baja atención que puede ser debida tanto a la dificultad que entraña su estudio taxonómico (Campbell, 1989; Deserti \& Zamponi, 2011, 2012) como por una cierta dificultad en localizar poblaciones. Ya sea por su escasez y baja densidad en los hábitats que ocupan, su pequeño tamaño y aspecto críptico, sus ciclos vitales a veces efímeros a lo largo de las estaciones climáticas, o bien por la dificultad de su localización en aguas profundas de lagos (de Moustier, 1950; Bryden, 1952; Ribi et al., 1985; Nalepa et al., 1987).

Jankowski et al. (2008) incluyen la presencia en la Península Ibérica de 2 especies del género Hydra, de un total de 12-15 especies conocidas a nivel mundial, con 4-6 de distribución paleártica. No se han podido encontrar apenas referencias ecológicas, taxonómicas o corológicas en las aguas epicontinentales ibéricas sobre este grupo (Morales et al., 2017), siendo Margalef (1955) la única referencia anterior que documenta la existencia de hidras en aguas durienses. Por lo tanto, el objetivo principal de este trabajo es comprobar su presencia en aguas de la cuenca del Duero, la caracterización de sus hábitats, sustratos y periodos de gemación; y finalmente la descripción gráfica de 2 taxones poco conocidos.

\section{Material y métodos}

Se muestrearon tramos de media montaña de los ríos Tera, incluyendo las orillas del lago de Sanabria, y Negro en el noroeste de Zamora; y del río Carrión en el noreste de Palencia (Fig. 1). Las localizaciones geográficas se presentan en la Tabla 1, así como las principales características fisicoquímicas del agua y los sustratos sobre el que se encontraron las hidras. En aguas del lago de Sanabria se hicieron muestreos trimestrales en 2016 y 2017 para conocer las comunidades epibiontes sobre la vegetación sumergida en el litoral somero (hasta 1,8 m) mediante inspección visual detallada. El material fue recogido y mantenido en vivo para su análisis en laboratorio. Asimismo se recogieron muestras cortadas o arrancadas de vegetales en zonas más profundas (hasta $4 \mathrm{~m}$ ) como sustrato de posible arraigo de las hidras, que fueron conservadas en etanol $70 \%$. Las medidas batimétricas se hicieron utilizando una ecosonda Hawkeye H22PX desde una zodiac en superficie. En el Negro y Carrión no se hicieron muestreos sistemáticos, aunque sí dirigidos a detectar invertebrados no artrópodos, en el marco de trabajos amplios de caracterización de la biodiversidad acuática. Todos los puntos de muestreo se corresponden con aguas frías y oligotróficas, muy transparentes y de muy escasa mineralización (Tabla 1).

Para denominar los taxones se utiliza la nomenclatura de Fauna Ibérica (Iberfauna, 2008) y GBIF.org (2018). Se determinaron atendiendo a claves dicotómicas, láminas y reseñas taxonómicas de Annandale (1911), Kramp (1935), Lee \& Campbell (1979), Campbell (1989), Holstein \& Emschermann (1995), Stepanjants et al. (2006), Hemmrich et al. (2007) y Schuchert (2010, 2012).

Algunos animales de taxones poco conocidos fueron mantenidos dentro de acuarios con aireación forzada, con agua y sustrato recogidos en las localidades estudiadas, bajo condiciones de temperatura y luz ambiente exterior. Se mantuvieron durante un breve periodo de tiempo (unas $48 \mathrm{~h}$, excepto el ejemplar comentado en resultados) únicamente con el objetivo de su correcta observación y documentación gráfica; así como poder obtener tejido fresco de tentáculos portadores de células con carácter taxonómico. 


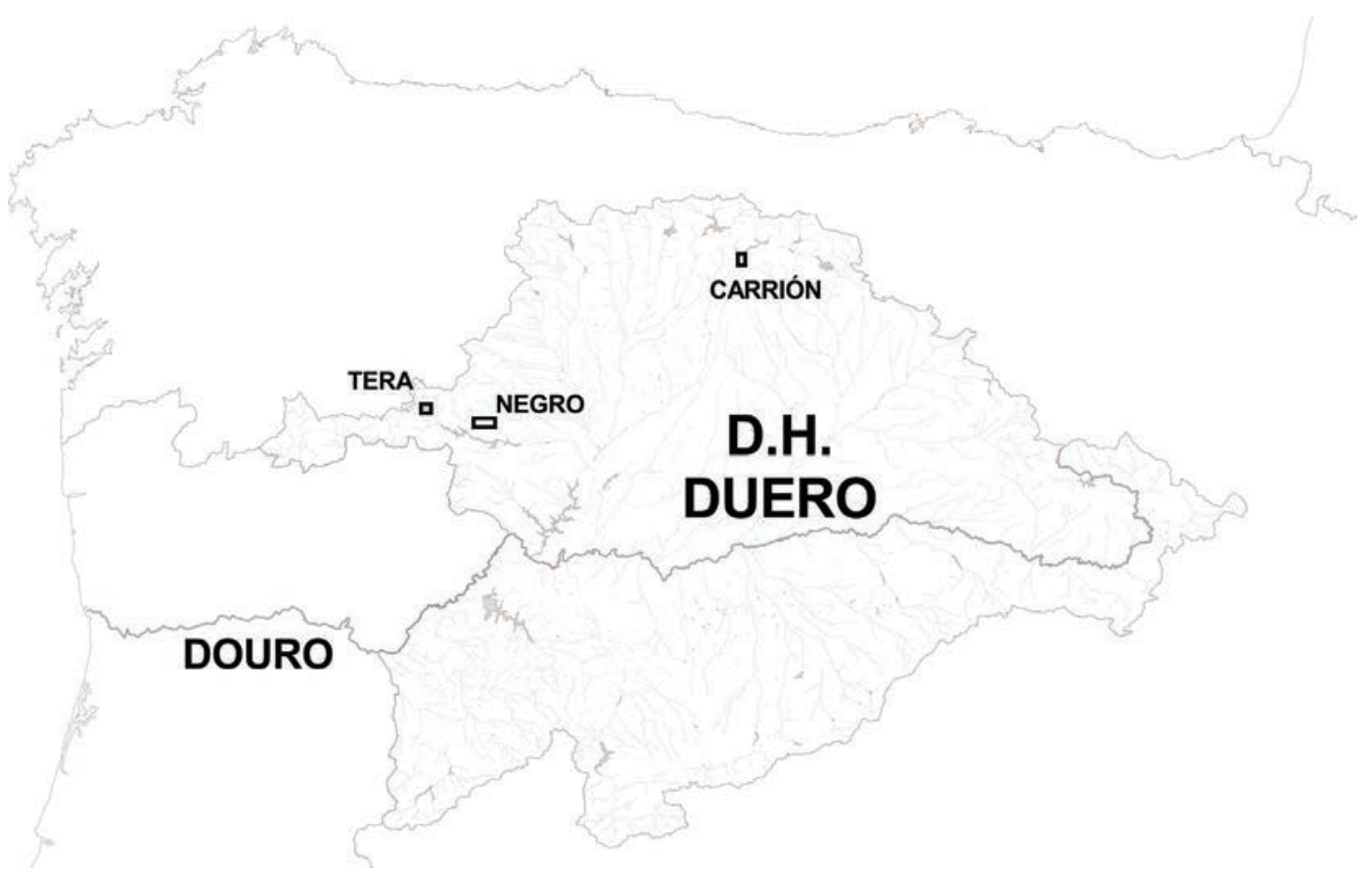

Fig. 1.- Localización de las tres áreas de estudio en el noroeste de la Península lbérica.

Fig. 1. - Location of the three study area in the northwest of the lberian Peninsula.

Tabla 1.- Localización geográfica, propiedades fisicoquímicas del agua [pH, COND: conductividad eléctrica, Tta: temperatura del agua (mes y año), oxígeno disuelto $\left(\mathrm{OD}, \% \mathrm{O}_{2}\right)$, sólidos totales disueltos (TDS)] y tipos de microhábitats sustrato donde se han encontrado los taxones en Zamora (ZA) y Palencia (PA). * Ecotipos según Anexo Il de Directiva Marco del Agua. 06: lago de media montaña, profundo, aguas ácidas; 25: ríos de montaña húmeda silícea.

Table 1.- Geographic location, physicochemical properties of the water [pH, COND: electrical conductivity, Tta: water temperature (month and year), dissolved oxygen $\left(\mathrm{OD}, \% \mathrm{O}_{2}\right)$, total dissolved solids (TDS)] and habitat types of microhabitats where taxa present in the provinces of Zamora (ZA) and Palencia (PA). * FWD Anex II ecotype. / mid-mountain 06: lake, deep, acidic Waters; 25: siliceous wet mountain rivers.

\begin{tabular}{|c|c|c|c|c|}
\hline Subcuenca: & Lago de Sanabria & río Tera (lago de Sanabria) & río Negro & río Carrión \\
\hline Localización: & ZA & ZA & ZA & PA \\
\hline \multirow[t]{2}{*}{ coordenadas: } & $\begin{array}{l}X: 42^{\circ} 06^{\prime} 50^{\prime \prime} \mathrm{N} \\
Y: 6^{\circ} 43^{\prime} 53^{\prime \prime} \mathrm{O} \\
\mathrm{Z}: 1000 \mathrm{msm}\end{array}$ & $\begin{array}{l}\mathrm{X}: 42^{\circ} 07^{\prime} 13^{\prime \prime} \mathrm{N} \\
\mathrm{Y:} 6^{\circ} 41 \text { '51"O } \\
Z: 995 \mathrm{msm}\end{array}$ & $\begin{array}{l}42^{\circ} 04^{\prime} 31^{\prime \prime} \mathrm{N} \\
6^{\circ} 21^{\prime} 18^{\prime \prime} \mathrm{O} \\
859 \mathrm{msm}\end{array}$ & $\begin{array}{l}42^{\circ} 51^{\prime} 09^{\prime \prime} \mathrm{N} \\
4^{\circ} 50^{\prime} 01^{\prime \prime O} \\
1160 \mathrm{msm}\end{array}$ \\
\hline & (nov 16) & & (ago 16) & $(\operatorname{sep} 14)$ \\
\hline Tipología de aguas: $\mathrm{pH}$ & 6,8 & & 5.6 & 7.5 \\
\hline $\operatorname{COND}(\mu \mathrm{S} / \mathrm{cm})$ & 9 & & 17 & 61 \\
\hline $\mathrm{OD}(\mathrm{mg} / \mathrm{l}) ; \% \mathrm{O}_{2}$ & 7,$01 ; 91,2$ & & 7,$82 ; 94$ & 10,$35 ; 110,5$ \\
\hline TDS $(\mathrm{mg} / \mathrm{l})$ & 4 & & 9 & 30 \\
\hline Tta $\left({ }^{\circ} \mathrm{C}\right):$ & \multicolumn{3}{|c|}{ 23,3 (sep 16), 13,2 (abr 18) } & 12,3 \\
\hline Dinámica: & lenítica: lago & lótica: rápidos, pozas & lótica: rápidos & lótica: rápidos \\
\hline Ecotipo DMA * & 06 & 25 & 25 & 25 \\
\hline Sustrato: & $\begin{array}{c}\text { macrófitas } \\
\cdot \text { Myriophyllum alterniflorum + Ranunculus } \\
\text { peltatus/pseudofluitans }+ \text { Potamogeton natans } \\
\cdot \text { Littorella uniflora+lsoetes velatum }+ \\
\text { Equisetum sppl } \cdot \text { Nitella flexilis }\end{array}$ & $\begin{array}{c}\text { macrófitas y hojarasca } \\
\cdot \text { M. alterniflorum+ } \\
\text { R. peltatus/pseudofluitans+ } \\
\text { P. natans }\end{array}$ & $\begin{array}{l}\text { macrófitas } \\
\cdot \text { M. } \\
\text { alterniflorum+ } \\
\text { R. peltatus/ } \\
\text { pseudofluitans }\end{array}$ & $\begin{array}{l}\text { rocoso } \\
\text {. cuarcita }\end{array}$ \\
\hline Hydra vulgaris Pallas, 1766 & $\mathrm{SI}$ & - & - & - \\
\hline $\begin{array}{l}\text { Hydra vulgaris var. } \\
\text { aurantiaca Ehrenberg, } 1838\end{array}$ & - & - & - & SI \\
\hline $\begin{array}{l}\text { Hydra (Pelmatohydra) } \\
\text { oligactis Pallas, } 1766\end{array}$ & $\mathrm{SI}$ & SI & - & - \\
\hline $\begin{array}{l}\text { Hydra (Chlorohydra) } \\
\text { viridissima Pallas, } 1766\end{array}$ & - & $\mathrm{SI}$ & $\mathrm{SI}$ & - \\
\hline
\end{tabular}


Pasado este tiempo los ejemplares murieron y se degeneraron sus tejidos, o bien fueron fijados en etanol $70 \%$. Se tomaron las medidas biométricas de partes de tentáculos y columna (Tabla 2) con un estereomicroscopio Nikon-SMZ800 de ocular micrométrico (10x). Las microfotografías del cnidoma se tomaron mediante aplastamientos (squash) en un microscopio invertido Nikon-EclipseTi equipado con software de medidas NIS Elements ${ }^{\circledR N i k o n ; ~ e n ~ c a d a ~ f o t o g r a f i ́ a ~}$ se indica la magnificación (Fig. 2 y 4) y se utilizó la técnica de contraste diferencial de interferencia (DIC) para algunas microfotografias del cnidoma. Las medidas de los cuatro tipos de cnidocitos (magnificación $\mathrm{x} 400$ ) se hicieron sobre preparaciones temporales de tejido fresco recién fijado en etanol $70 \%$, y con las cápsulas sin descargar.

\section{Resultados}

Se han recogido datos de la presencia de cuatro taxones (Tabla 1) en aguas de la cuenca del Duero, en tres subcuencas de su vertiente norte. En todos los tramos de muestreo, localizadas en zonas de piedemonte, se localizó un único taxón. Si bien en el lago de Sanabria se encontraron 3 especies, aunque distribuidas en simpatría. En el río Carrión se localizaron dos pequeños grupos de hidra común de pigmentación anaranjada, Hydra vulgaris var. aurantiaca Ehrenberg, 1838 adheridas a bloques de cuarcita sumergidos en una zona de fuerte corriente bajo la presa de Compuerto (Tabla 1). Compuestos por 8 y 12 ejemplares que se encontraban en gemación (Fig. 2). La observación se realizó en septiembre de 2014, y con posterioridad no fueron localizados nuevos ejemplares en los siguientes muestreos del otoño. Los otros tres taxones se localizaron en la subcuenca del Tera, tanto en aguas del lago de Sanabria, como en el tramo medio del río Negro (Tabla 1).

En tramos someros del lago, con amplia cobertura de plantas macrófitas sumergidas y helófitas, se localizaron durante el verano ejemplares de Hydra vulgaris Pallas, 1766 que presentaron la típica coloración ocre-marrón, algo hialina. Se hallaron sobre tallos vivos sumergidos hasta $6 \mathrm{~m}$ de profundidad (Tabla 1). Además, en las pozas de salida del río Tera desde el lago se capturó un ejemplar solitario de Hydra (Pelmatohydra) oligactis Pallas, 1766, sobre vegetación y hojarasca en descomposición en el mes de noviembre. Esta misma especie se detectó de nuevo sobre praderas sumergidas de Nitella flexilis (Linnaeus) C. Agardh 1824, en fondos de sustrato arenoso y formando pequeños grupos (Fig. 3A). En el mismo tramo de salida del Tera, zona muy soleada
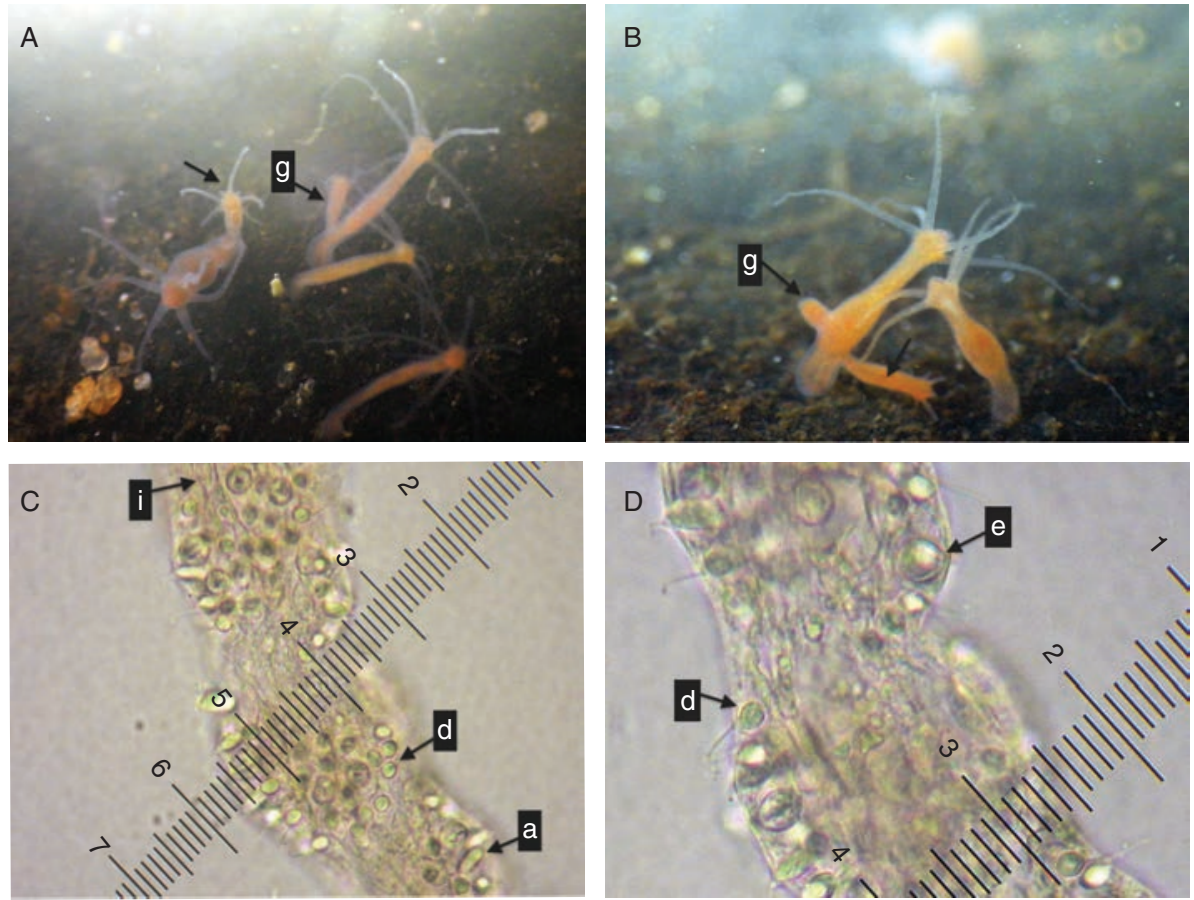

Fig. 2.- Galería fotográfica de Hydra vulgaris var. aurantiaca. A-B: aspecto "in situ" de la pequeña colonia en su hábitat bentónico (flechas negras señalan yemas y pólipos en desarrollo por gemación, g) sobre una cuarcita sumergida dentro de un rápido del río Carrión. C: tejidos del brazo, con disposición de los nematocistos agrupados (x200). D: detalle ampliado de un grupo de nematocistos (x400), algunos disparados con el cnidocilio al exterior. Nematocistos: e, estenoteles; d, desmonemas; a, isorhizas atricas; $h$, isorhizas holotricas. Magnificación x200: 1 unidad de regleta $=5 \mu \mathrm{m} ; \times 400: 2,5 \mu \mathrm{m}$.

Fig. 2.- Gallery of Hydra vulgaris var. aurantiaca. A-B: aspects of the small colony in its benthic habitat (black arrows indicate buds and polyps in development by budding, g) on a quartzite submerged inside on the river Carrión. C: tissues of the arm, with disposition of the grouped nematocysts (x200). D: enlarged detail of a group of nematocysts (x400), some shot with the cnidocylia outside. Nematocysts: (e) stenoteles, (d) desmonemes, (a) atrichous isorhizas, (i) holotrichous isorhizas. Magnification x200: 1 bar unit $=5 \mu \mathrm{m} ; x 400: 2,5 \mu \mathrm{m}$. 
de fondos de gravas y arenas cubierta por milenramas y ranúnculos, se localizó durante el verano Hydra (Chlorohydra) viridissima Pallas, 1766 (Tabla 1). Esta misma especie fue localizada sobre estas mismas plantas en el río Negro, y también en verano; siendo en ambos casos ejemplares completamente verdes (Tabla 2) y teniendo como soporte tallos vivos.

Un ejemplar de $H$. oligactis se mantuvo durante 7 días en noviembre en acuario a temperatura ambiente, conservando el resto de parámetros del agua ( $\mathrm{pH}$ y oxígeno disuelto) próximos a los ambientales (Tabla 1).
Durante este periodo se pudo documentar gráficamente su movimiento en la cámara de observación al microscopio, así como su comportamiento depredador sobre un gusano oligoqueto Stylodrilus heringianus Claparede, 1862 que formaba parte de la misma comunidad bentónica de la hojarasca (Fig. 3). Esta hidra marrón mostró en cautividad un comportamiento lucífugo acusado, aunque de movimientos vigorosos durante su movilidad.

En la Tabla 2 se presentan las medidas biométricas tomadas sobre ejemplares vivos en reposo, así como de
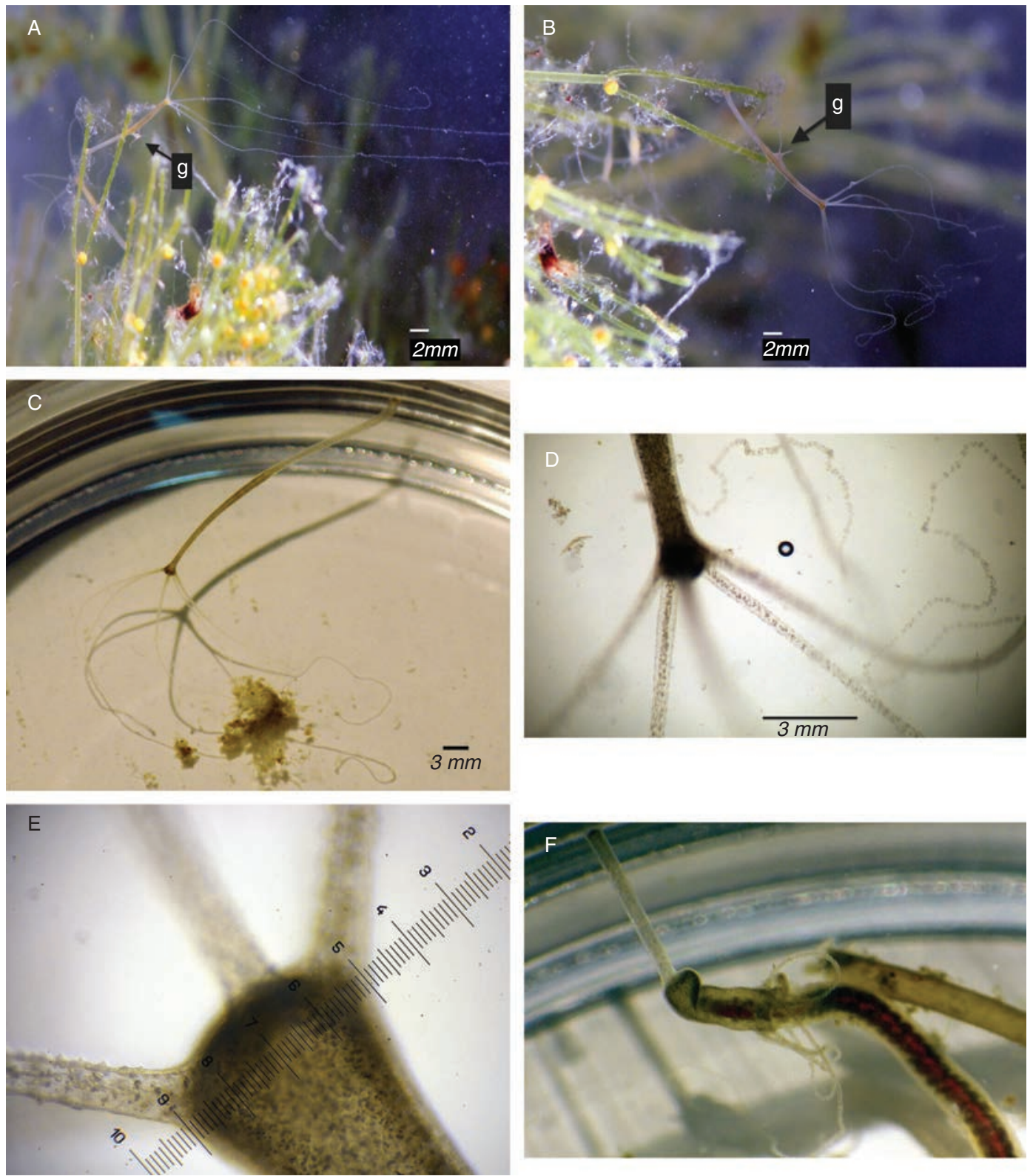

Fig. 3.- Fotografías de detalle de Hydra oligactis. A-B: un grupo de hidras sobre Nitella flexilis, en acuario (g: gemación). C: habitus de un ejemplar en la cámara de observación. D: aspecto del animal activo con los tentáculos desplegados. E: detalle del hipostoma y los tejidos de la parte anterior de la columna. F: un gusano oligoqueto (Stylodrilus heringianus) parcialmente introducido en la cavidad gastrovascular. Magnificación (x200) como en Fig 2.

Fig. 3.- Detail pictures of Hydra oligactis. A-B: a group of hydras on Nitella flexilis, in aquarium (g: budding). C: habitus of a specimen in the observation chamber. D: appearance of the active animal with the tentacles unfolded. E: detail of the hypostoma and the tissues of the anterior part of the columna. F: an oligochaete worm (Stylodrilus heringianus) partially introduced in the coelentereon. Magnification (x200) as in Fig 2. 
Tabla 2.- Características morfológicas de ejemplares observados "in vivo" $\left(\mathrm{N}_{\mathrm{v}}\right)$ y biometría básica (microscopio óptico) de los ejemplares medidos $(N)$ en cautividad de ambas especies de hidras y sus nematocistos (L: longitud, $A$ : anchura, $n$ : número de células). (Valores promedio \pm desviación estándar, [rango]).

Table 2.- Morphological characteristics of specimens observed "in vivo" $\left(\mathrm{N}_{\mathrm{v}}\right)$ and basic biometry (optical microscope) of the specimens measured in captivity $(\mathrm{N})$ of both hydras species and their nematocysts (L: length, $\mathrm{A}$ : width, $\mathrm{n}$ : number of cells). (Mean values \pm standard deviation, [range]).

\begin{tabular}{|c|c|c|c|c|c|c|}
\hline & \multicolumn{3}{|c|}{$\begin{array}{l}\text { Hydra vulgaris var. aurantiaca } \\
\left(\mathrm{N}_{\mathrm{v}}=15\right)\end{array}$} & \multicolumn{3}{|c|}{$\begin{array}{l}\text { Hydra (Pelmatohydra) oligactis } \\
\left(\mathrm{N}_{\mathrm{v}}=9\right)\end{array}$} \\
\hline $\mathrm{N}^{\circ}$ brazos & \multicolumn{3}{|c|}{6} & \multicolumn{3}{|c|}{5} \\
\hline $\mathrm{N}^{\circ}$ yemas o pólipos & \multicolumn{3}{|c|}{$[0-2] ;(N=6)$} & \multicolumn{3}{|c|}{$[0-1] ;(N=5)$} \\
\hline Color del cuerpo & \multicolumn{3}{|c|}{ rojo-anaranjado } & \multicolumn{3}{|c|}{ gris } \\
\hline Diámetro pie $(\mathrm{mm}) \mathrm{DP}$ & \multicolumn{3}{|c|}{$2.2 \pm 0.2 ;(\mathrm{N}=3)$} & \multicolumn{3}{|c|}{1.61} \\
\hline Diámetro columna_base $(\mathrm{mm}) \mathrm{DCB}$ & \multicolumn{3}{|c|}{$2.6 \pm 0.3 ;(N=3)$} & \multicolumn{3}{|c|}{1.64} \\
\hline Diámetro columna_máx. $(\mathrm{mm}) \mathrm{DCM}$ & \multicolumn{3}{|c|}{$2.8 \pm 0.3 ;(N=3)$} & \multicolumn{3}{|c|}{2.24} \\
\hline Longitud columna_máx. (mm) LCM & \multicolumn{3}{|c|}{$8.9 \pm 0.6 ;(N=3)$} & \multicolumn{3}{|c|}{12.2} \\
\hline Longitud brazos_máx. (mm) LBM & \multicolumn{3}{|c|}{$11.9 \pm 0.6 ;(N=3)$} & \multicolumn{3}{|c|}{27.2} \\
\hline Diámetro hipostoma $(\mathrm{mm}) \mathrm{DH}$ & \multicolumn{3}{|c|}{$3.0 \pm 0.4 ;(N=3)$} & \multicolumn{3}{|c|}{2.1} \\
\hline Nematocistos $(\mu \mathrm{m})$ : & L & A & L/A & L & $A$ & L/A \\
\hline estenoteles (e) & $15.4 \pm 1.2$ & $13.1 \pm 1.5$ & $1.18 \pm 0.04(n=11)$ & $12 \pm 0.5$ & $6.9 \pm 0.5$ & $1.74 \pm 0.05(n=7)$ \\
\hline desmonemes (d) & $7.3 \pm 0.2$ & $4.9 \pm 0.6$ & $1.51 \pm 0.18(n=9)$ & $5.3 \pm 0.7$ & $4.0 \pm 0.4$ & $1.33 \pm 0.13(n=6)$ \\
\hline isorhizas atricas (a) & $7.1 \pm 0.1$ & $4.3 \pm 0.2$ & $1.65 \pm 0.07(n=5)$ & $7.7 \pm 1.6$ & $3.9 \pm 0.7$ & $1.99 \pm 0.16(n=5)$ \\
\hline isorhizas holotricas $(\mathrm{h})$ & $11.4 \pm 0.6$ & $4.5 \pm 0.3$ & $2.50 \pm 0.08(n=9)$ & $8.7 \pm 0.9$ & $4.0 \pm 0.4$ & $2.15 \pm 0.12(n=5)$ \\
\hline
\end{tabular}

las células de su cnidoma. H. oligactis se corresponde con una especie estilizada y flexible, capaz de extender el cuerpo y los tentáculos 3.05 veces su talla $(\mathrm{LC}=12$ $\mathrm{mm}$ ); y con los cnidocitos muy dispersos y poco abundantes entre los nudos de los tentáculos, que completamente extendidos alcanzan $37.2 \mathrm{~mm}$. A la vez la columna está diferenciada en dos mitades, siendo la basal de diámetro casi idéntico al pie y la parte distal que contiene la cavidad gastrovascular más ancha; con una relación $\mathrm{DCB} / \mathrm{DP}=1.02$ y $\mathrm{DCM} / \mathrm{DP}=1.37$. Mientras que $H . v$. aurantiaca presentó un aspecto mucho más grueso en la columna ( $\mathrm{LC}=8.9 \mathrm{~mm}$ y tentáculos 1,3 veces esta longitud) con un diámetro similar a lo largo $\mathrm{DCB} / \mathrm{DP}=$ 1.18 y $\mathrm{DCM} / \mathrm{DP}=1.08$. La primera especie presenta un hipostoma de cinco tentáculos $\left(\mathrm{N}_{\mathrm{V}}=1\right)$, más ancho que la base del pie $(\mathrm{DH} / \mathrm{DP}=1.30)$; y la segunda de ancho muy similar, con seis tentáculos $\left(\mathrm{N}_{\mathrm{V}}=15\right)$. Estenoteles e isorhizas holotricas son los cnidocitos más diferentes entre ambas especies comparadas (Tabla 2).

\section{Discusión}

Nuestros resultados amplían el espectro de especies recogidas por Jankowski et al. (2008) para la zona noroccidental de la Península Ibérica, para la que indica sólo 2 taxones. De esta manera son cuatro los integrantes de la hydridofauna en estos ríos, al igual que indican para la red fluvial de Centroeuropa.

El lago de Sanabria como ecosistema de referencia ecológica del NO ibérico es el punto de mayor riqueza con 3 especies presentes, a lo que puede contribuir el régimen térmico de la masa de agua que permite aguas algo más cálidas en otoño (Morales et al., 2017). Es conocido en lagos de origen glaciar como el de Sanabria la presencia de especies de hidras (de Moustier, 1950; Ribi et al., 1985); incluso en aguas muy profundas, a más de $300 \mathrm{~m}$ de la superficie (Nalepa et al., 1987). Nosotros hemos encontrado ejemplares hasta una profundidad de $6,5 \mathrm{~m}$, sobre el nivel de cota máxima, mientras que Ribi et al. (1985) describen la zona eufótica (3-17 m) de los lagos Zürich y Maggiore como la preferida por las hidras.

Por otro lado, se documenta en este trabajo la presencia en la Península Ibérica de la hidra marrón (H. oligactis); una especie de amplia distribución por el hemisferio norte y parte de Australia. Su preferencia por orillas someras con abundante vegetación acuática y raíces sumergidas hace habitual su presencia en aguas no contaminadas; siendo sus hábitats preferidos pozas de arroyos, lagunas y lagos templados. En las orillas del lago de Sanabria se encontraron durante el otoño en remansos muy someros de fondos cubierto por raíces de los árboles de ribera, y hojarasca en descomposición recién caída al agua. En primavera se localizaron sobre praderas sumergidas de Nitella flexilis (Fig. 3A-B); momento en que se detectó gemación. Se localizaron tanto ejemplares aislados como grupos de 3-5 ejemplares; aunque Morpurgo (2017) encuentra grupos de hasta 25 ejemplares en el lago Grande de Monticolo.

Poco se conoce sobre la ecología de las especies del género Hydra en el medio natural ibérico. Las hidras, especialmente en primavera, parece que muestran tendencia a frecuentar las proximidades de briozoos y poríferos (Morpurgo, 2017); como Spongilla lacustris 
(Linnaeus, 1758), frecuente en nuestros puntos de muestreo. Annandale (1911) señala que posiblemente se debe al refugio que les proporcionan.

Las hidras deben tener un papel importante como predadores en las comunidades bentónicas, aun por estudiar; y parecen tener pocos enemigos naturales. Annandale (1911) describe en ejemplares de $H$. vulgaris cautivos en estanques su predación habitual por larvas de mosquitos. Además recoge el ataque sobre $H$. vulgaris "rubra" en lagos escoceses del gusano platelminto Microstomum lineare Örsted, 1843. En su papel como predadores en las comunidades bentónicas Schwartz et al. (1983) señalan a los crustáceos del plancton como sus presas principales, así como a los gusanos oligoquetos. Deserti et al. (2017) recogen la presencia de un amplio espectro de micro-invertebrados, hongos y microalgas en la cavidad gastrovascular de $H$. vulgaris. El ataque de $H$. oligactis sobre un oligoqueto se muestra en la Fig. 3.
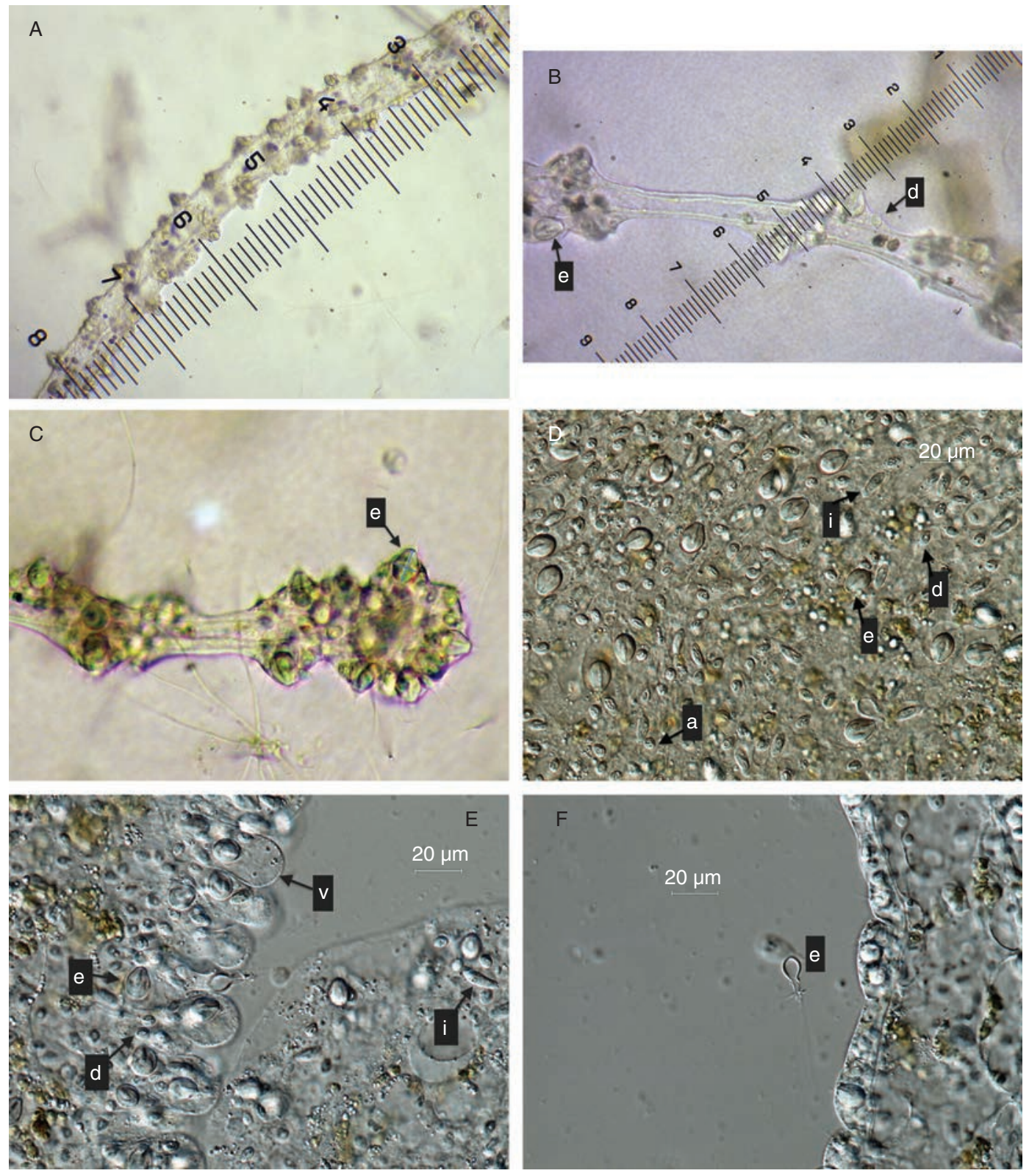

Fig. 4.- Microfotografías del cnidoma de Hydra oligactis. A: extremo de un tentáculo durante la fase de actividad, con grupos de nematocistos en los nudos. B: detalle completamente extendido sin ellos en los internados. C: nematocistos agrupados en el extremo del tentáculo. D: grupos de células de la epidermis columnar obtenidos mediante la técnica de "squash". E: distintos tipos de cnidocitos (células mucosas y enzimáticas) en el endodermo de la cavidad gastrovascular (v) y nematocistos de la columna. F: detalle de una cápsula (c) con estiletes, disparada en un estenotele. Aumentos: A, x200; B-C, x400; D-F, x400-DIC. Abreviaturas como en Fig. 2.

Fig. 4.- Microphotographs of Hydra oligactis cnidoma. A: end of a tentacle during the activity phase, with groups of nematocysts at the nodes. B: detail fully extended without them at the internotes. C: nematocysts grouped at the end of the tentacle. D: groups of cells of the columnar epidermis with "squash" technique. E: different types of cnidocytes (mucosal and enzymatic cells) at the coelentereon (v) and nematocysts of columna. F: detail of a capsule (c) with stylets, shot into a stenotele. Magnification: A, x200; B-C, x400; D-F, x400-DIC. Abbreviations as in Fig. 2. 
Los tramos del Tera y Negro donde se han localizado las hidras son habitados en sintopía por comunidades de otras especies bentónicas consideradas como indicadores de buen estado ecológico para los ecosistemas de aguas frías, ácidas, oligotróficas y oligosaprobias (Tachet et al., 2000). Como es el caso de Margaritifera margaritifera (Linnaeus, 1758), Spongilla lacustris y Heteromeyenia baileyi (Bowerbank, 1863) o las algas rojas Lemanea fluviatilis (L.) C. Agardh y Volatus carrionii I.S. Chapuis \& Vis, 2017; presentes en ambos cauces zamoranos (Chapuis et al., 2017; Morales et al., 2017). Por otro lado las poblaciones de H. vulgaris del río Carrión están sometidas a un régimen térmico inverso debido a su localización aguas debajo del pantano de Compuerto, que libera abundante caudal del hipolimnion durante el verano lo cual impide alcanzar la temperatura habitual de los ríos en esta estación e imposibilita en gran medida la reproducción de otros invertebrados (Flechoso et al., en prensa).

En este sentido Mooney et al. (2018) comprueban como las conclusiones de los bioensayos con $\mathrm{H}$. viridissima en la relación $\mathrm{pH} /$ toxicidad del amonio, para el establecimiento de las directrices de calidad (USEPA, 2013) deben revisarse. Ya que en realidad la sensibilidad de esta especie a la presencia de amonio libre, en ambientes reductores de $\mathrm{pH}$ elevado, depende del grado de mineralización del agua, y no sólo del efecto combinado de la toxicidad y la temperatura. De manera que podría aguantar un nivel de toxicidad crónica del amonio mayor de lo esperado en aguas ácidas.

Otra característica que ha atraído a los científicos sobre este grupo de cnidarios es su plasticidad fenotípica como respuesta a los cambios ambientales y la toxicidad (Huarachi \& González, 2012). En este sentido Deserti et al. (2010) encuentran cnidomas diferentes para $H$. vulgaris en lagunas próximas pero con diferentes calidades ambientales. En los ejemplares del Tera se encontraron únicamente isorhizas holotricas del Tipo II , es decir con forma de semilla; y no la forma de paramecio o zapatilla del Tipo I, que los autores relacionan con aguas menos transparentes y algo eutróficas.

Mediante secuenciación de ADN mitocondrial y nuclear Martínez et al. (2010) definen cuatro grupos de especies distribuidas por todo el Planeta, en especial las hidras verdes y las hidras marrón-gris del grupo "vulgaris" que son cosmopolitas. Siendo de ámbito reducido al hemisferio holártico las hidras grises de los grupos "oligactis" y "braueri"; que incluye a $H$. circumcincta, presente en España pero que no ha sido localizada en la cuenca del Duero. Schwentner \& Bosch (2015) concluyen que esta distribución podría haber sido muy alterada por transporte antrópico hacia el hemisferio sur, si bien dada su larga historia evolutiva son también capaces de dispersarse a grandes distancias. En muchos casos la plasticidad genotípica produce variaciones morfológicas, incluidos corotipos desarrollados en ámbitos geográficos particulares, que dificultan su clasificación taxonómica por lo que es necesario profundizar en el conocimiento de este grupo y completar la información corológica en la Península Ibérica.

\section{Agradecimientos}

Los trabajos se desarrollaron parcialmente dentro del proyecto de investigación Caracterización de la biodiversidad en Velilla del Río Carrión (2013-14), financiado por la Cátedra Iberdrola de la Universidad de Salamanca, y también al Programa de Seguimiento Limnológico Bianual Intensivo del Lago de Sanabria (2016-17) financiado por Confederación Hidrográfica del Duero. Se tuvieron en cuenta las anotaciones de dos revisores para mejorar el manuscrito original.

\section{Referencias}

Annandale, N., 1911. The fauna of British India including Ceylon and Burma. Freshwater sponges, Hydroids \& Polyzoa. Taylor and Francis. London. viii +251 pp. https://doi.org/10.5962/bhl.title. 1050

Beach, M. J. \& Pascoe, D., 1998. The role of Hydra vulgaris (Pallas) in assessing the toxicity of freshwater pollutants. Water Research, 32(1): 101-106. https://doi. org/10.1016/S0043-1354(97)00180-2

Bode, H. R., 2003. Head regeneration in Hydra. Developmental Dynamics, 226: 225-236.

Bryden, R. R., 1952. Ecology of Pelmatohydra oligactis in Kirkpatricks Lake, Tennessee. Ecological Monographs, 22: 45-68. https://doi.org/10.2307/1948528

Campbell, R. 1989. Taxonomy of the European Hydra (Cnidaria: Hydrozoa): a re-examination of its history with emphasis on the species $H$. vulgaris Pallas, $H$. attenuata Pallas and H. circurncincta Schulze. Zoological Journal of the Linnean Society, 95: 219-244. https://doi. org/10.1111/j.1096-3642.1998.tb01992.x

CCME (Canadian Council of Ministers of the Environment). 2010. Canadian water quality guidelines for the protection of aquatic life: Ammonia. In: Canadian environmental quality guidelines, 1999. Canadian Council of Ministers of the Environment. Winnipeg: 1-8.

Chapuis, I. S., Necchi, O. Jr., Zuccarello, G. C., Xie, S. L., Aboal, M., Sánchez-Castillo, P. M. \& Vis, M. L., 2017. A new genus, Volatus and four new species of Batrachospermum sensu strict (Batrachospermales, Rhodophyta). Phycologia, 56(4): 454-468. https://doi. org/10.2216/16-73.1

de Moustier, J. M., 1950. Le Lac de Constance. Bulletin Français de la Pêche et de la Pisciculture, 159: 56-70. https://doi.org/10.1051/kmae:1951013

Deserti, M. I., Esquius, K. S., Escalante, A. H. \& Acuña, F. H., 2017. Trophic ecology and diet of Hydra vulgaris (Cnidaria; Hydrozoa). Animal Biology, 67(3-4): 287-300. https://doi.org/10.1163/15707563-00002537

Deserti, M. I. \& Zamponi, M. O., 2011. Hydra vulgaris Pallas, 1766, (=Hydra attenuata) (Cnidaria; Hydrozoa) from the Los Padres Lagoon (Buenos Aires province, Argentina). Revista Brasileira de Biociências, 9(4): 482-487. http://www.ufrgs.br/seerbio/ojs/index.php/ rbb/article/view/1753 
Deserti, M. I. \& Zamponi, M. O., 2012. Biometric and statistical investigations on the cnidoma of the genus Hydra (Cnidaria, Hydrozoa). Iheringia, Série Zoologia, 102(3): 298-302. https://doi.org/10.1590/S007347212012000300008

Deserti, M. I., Zamponi, M. O. \& Escalante A. H., 2010. Dos tipos del nematocisto, holotrico isorriza, en Hydra vulgaris Pallas , 1766 (Cnidaria, Hydrozoa) de diferentes aguas continentales. Revista Real Academia Galega de Ciencias, 29: 67-76.

Deserti, M. I., Zamponi, M. O. \& Escalante, A. H., 2011. The genus Hydra from Argentina. I. Hydra vulgaris pedunculata subsp. nov. (Cnidaria, Hydrozoa). Revista Real Academia Galega de Ciencias, 30: 5-14.

Flechoso, F., Morales, J. \& Lizana, M. (en prensa). Relación entre la temperatura y régimen hidráulico con la diversidad de odonatos en el río Carrión (Palencia, España). Boletín de la Sociedad Entomológica Aragonesa.

GBIF, 2018. GBIF Home Page. Available at https://www. gbif.org [accesed at 07/05/2018].

Ghaskadbi, S. S., Shetye, L., Chiplonkar, S. \& Ghaskadbi, S., 2005 Ultraviolet irradiation initiates ectopic foot formation in regenerating hydra and promotes pudding. Journal of Biosciences, 30: 177-182. https://doi. org/10.1007/BF02703697

Holstein, T. \& Emschermann, P., 1995. Sübwasserfauna von Mittteleuropa, Band 1/2+3. Cnidaria: Hydrozoa, Kamptozoa. Fischer Verlag. 141 pp.

Hemmrich, G., Anokhin, B., Zacharias, H. \& Bosch, T. C. G., 2007. Molecular phylogenetics in Hydra, a classical model in evolutionary developmental biology. Molecular Phylogenetics and Evolution, 44: 281-290. https:// doi.org/10.1016/j.ympev.2006.10.031

Huarachi, R. \& González, R., 2012. Hydra vulgaris Pallas, 1766 (Hydrozoa: Hydridae) como bioindicador de la calidad de aguas del río Chili, Arequipa, Perú. The Biologist (Lima), 10(2): 125-137.

Iberfauna, 2008. Genus Hydra. Linnaeus, 1758. En: IBERFAUNA. El Banco de Datos de la Fauna Ibérica. Museo Nacional de Ciencias Naturales (CSIC). http://iberfauna. mncn.csic.es/showficha. aspx rank $=\mathrm{T} \& \mathrm{idtax}=28656$ [accessed 07/05/2018].

Jankowski, T. \& Collins, A. C. \& Campbell, R. D., 2008. Global diversity of inland water cnidarians. Hydrobiologia, 595: 35-40. https://doi.org/10.1007/ s10750-007-9001-9

Kar, S. \& Aditya A. K., 2007. Evaluation of freshwater toxicity with Hydra as a test animal. Philippine Journal of Science, 136 (2): 173-179.

Khalturin, K., Anton-Erxleben F., Sassmann S., Wittlieb J., Hemmrich, G. \& Bosch T., 2008. A novel gene family controls species-specific morphological traits in Hydra. PLoS Biology, 6(11): e278. https://doi.org/10.1371/ journal.pbio.0060278

Kaliszewicz, A., 2011. Interference of asexual and sexual reproduction in the green hydra. Ecological Resources, 26: 147-152. https://doi.org/10.1007/s11284-010-0771-6

Kaliszewicz, A. \& Lipinska, A., 2013. Environmental condition related reproductive strategies and sex ratio in hydras. Acta Zoologica (Stockholm), 94: 177-183. https://doi.org/10.1111/j.1463-6395.2011.00536.x
Kramp, P. L., 1935. Polypdyr (Coelenterata). I Ferskvandspolypper og goplepolypper. Danmarks fauna, 41. Dansk Naturhistorisk Forening. 207 pp.

Lee, H. T. \& Campbell, R. D., 1979. Development and behavior of an intergeneric chimera of hydra (Pelmatohydra oligactis interstitial cells: Hydra attenuata epithelial cells). The Biological Bulletin, 157: 288-296. https://doi.org/10.2307/1541055

Margalef, R., 1955. Contribución al estudio de la fauna de aguas dulces del noroeste de España. Publicaciones del Instituto de Biología Aplicada, 31: 137-171.

Martínez, D. E. \& Bridge, D., 2012. Hydra, the everlasting embryo, confronts aging. The International Journal of Developmental Biology, 56: 479-487. https://doi. org/10.1387/ijdb.113461dm

Martínez, D. E., Iñiguez, A. R., Percella, K. M., Willner, J. B. Signorovitch, J. \& Campbell, R.D., 2010. Phylogeny and biogeography of Hydra (Cnidaria: Hydridae) using mitochondrial and nuclear DNA sequences. Molecular Phylogenetics and Evolution, 57(1): 403-410. https:// doi.org/10.1016/j.ympev.2010.06.016

Massaro, F. C. \& Rocha, O., 2008. Development and population growth of Hydra viridissima Pallas, 1766 (Cnidaria, Hydrozoa) in the laboratory. Brazilian Journal of Biology, 68(2): 379-383. https://doi.org/10.1590/ S1519-69842008000200020

Mooney, T. J., Pease, C., Trenfield, M., van Dam, R. \& Harford, A. J., 2018. Modeling the $\mathrm{pH}-$ ammonia toxicity relationship for Hydra viridissima in soft waters with low ionic concentrations. Environmental Toxicology and Chemistry, 37(4): 1189-1196. https://doi. org/10.1002/etc.4071

Morales, J., Negro, A. I. \& Lizana, M., 2017. Reseñas sobre diversidad de invertebrados no artrópodos dulceacuícolas en ecosistemas oligotróficos y poco mineralizados de la red Natura 2000 (NO de España). Nova Acta Cientifica Compostelana (Bioloxía), 24: 21-44.

Morpurgo, M., 2017. Presence of brown hydra Hydra oligactis Pallas, 1766 (Hydrozoa: Anthoathecata: Hydridae) in Large Lake of Monticolo (South Tyrol, Prov. Bolzano, Italy). Gredleriana, 17: 249-252.

Nalepa, T., Remsen, C. C \& Val Klump, J., 1987. Observations of Hydra from a submersible at two deepwater sites in lake Superior. Journal of Great Lakes Research, 13(1):84-87. https://doi.org/10.1016/ S0380-1330(87)71630-X

Quinn, B., Gagné, F. \& Blaise C., 2012. Hydra, a model system for environmental studies. The International Journal of Developmental Biology, 56: 613-625. https:// doi.org/10.1387/ijdb.113469bq

Rosa, M. E., Bradacs, S. \& Tökölyi, J., 2015. Response of green hydra (Hydra viridissima) to variability and directional changes in food availability. Biologia, 70(10): 1366-1375. https://doi.org/10.1515/biolog-2015-0161

Tökölyi, J., Bradács, F., Hóka, N., Kozma, N., Miklós, M., Mucza, O., Lénárt, K., Sebestyén, F. \& Barta, Z., 2016. Effects of food availability on asexual reproduction and stress tolerance along the fast-slow life history continuum in freshwater hydra (Cnidaria: Hydrozoa). Hydrobiologia, 766(1): 121-133. https:// doi.org/10.1007/S10750-015-2449-0 
Ribi, G., Tardent, R., Tardent, P. \& Scascighini, C., 1985. Dynamics of hydra populations in Lake Zurich, Switzerland, and Lake Maggiore, Italy. Swiss Journal of Hydrology, 47(1): 45-56. https://doi.org/10.1007/BF02538183

Schroeder, L. A. \& Callaghan, W. M., 1982. Effects of temperature on the energy budgets of Hydra pseudoligactis. Oecologia (Berlin), 53: 238-244. https://doi. org/10.1007/BF00545670

Schuchert, P., 2010. The European athecate hydroids and their medusae (Hydrozoa, Cnidaria): Capitata Part 2. Revue Suisse de Zoologie, 117(3): 337-555. https://doi. org/10.5962/bhl.part.117793

Schuchert, P., 2012. North-West European Athecate Hydroids and their Medusae. Synopses of the British Fauna (New Series), 59: 1-365.

Schwartz, S. S., Hann, B. J. \& Hebert, P. D. N., 1983. The feeding ecology of Hydra and possible implications in the structuring of pond zooplankton communities.
The Biological Bulletin, 164: 136-142. https://doi. org/10.2307/1541196

Schwentner, M. \& Bosch, T. C. G., 2015. Revisiting the age, evolutionary history and species level diversity of the genus Hydra (Cnidaria: Hydrozoa). Molecular Phylogenetics and Evolution, 91: 41-55. https://doi. org/10.1016/j.ympev.2015.05.013

Stepanjants, S. D., Anokhin, B. A \& Kuznetsova, V. G., 2006. Cnidarian fauna of relict Lakes Baikal, Biwa and Khubsugul. Hydrobiologia, 568: 225-232. https://doi. org/10.1007/s10750-006-0310-1

Tachet, H., Richoux, P., Bournaud, M. \& Usseglio-Polatera, P., 2000. Invertébrés d'eau douce: systématique, biologie, écologie. CNRS éditions. Paris. 588 pp.

USEPA (US Environmental Protection Agency). 2013. Aquatic life ambient water quality criteria for ammonia-Freshwater 2013. US Environmental Protection Agency. Washington, DC. 242 pp. 